\title{
Electric and magnetic properties of titanium-cobalt-oxide single crystals produced by floating zone melting with light heating
}

\author{
A.M. Balbashov ${ }^{1}$, A.A. Mukhin ${ }^{2}$, V.Yu. Ivanov ${ }^{2}$, L.D. Iskhakova ${ }^{3}$, and M.E. Voronchikhina ${ }^{1}$ \\ ${ }^{1}$ Moscow Power Engineering Institute, Moscow, Russia \\ E-mail: BalbashovAM@mpei.ru \\ ${ }^{2}$ Prokhorov General Physics Institute of RAS, Moscow, Russia \\ ${ }^{3}$ Fiber Optics Research Center of RAS, Moscow, Russia
}

Received January 2, 2017, published online June 26, 2017

\begin{abstract}
Single crystals of spinel $\mathrm{Co}_{2} \mathrm{TiO}_{4}, \mathrm{CoTiO}_{3}$ of ilmenite structure and pseudobrookite $\mathrm{CoTi}_{2} \mathrm{O}_{5}$ were grown by means of zone melting equipment URN-2-ZM. The growth processes were performed in an air atmosphere with a speed $\sim 10 \mathrm{~mm} / \mathrm{h}$ and a final annealing of the crystal at temperature of $1250{ }^{\circ} \mathrm{C}$. $\mathrm{Co}_{2} \mathrm{TiO}_{4}$ and $\mathrm{CoTi}_{2} \mathrm{O}_{5}$ crystals were obtained with a diameter of $12-15 \mathrm{~mm}$ and length up to $60 \mathrm{~mm}$, being free of any other phase inclusions and cracks. However, in the $\mathrm{CoTiO}_{3}$ crystals some controversial features were found: $\mathrm{x}$-ray Laue analysis indicated high-structure perfection while an electronic microscopy revealed small amount of second phase inclusions in contradiction to known phase diagram of this system. Electrical and magnetic properties of grown $\mathrm{Co}_{2} \mathrm{TiO}_{4}$ and $\mathrm{CoTiO}_{3}$ single crystals were studied. Semiconducting behavior of the $\mathrm{Co}_{2} \mathrm{TiO}_{4}$ was established with the energy gap of $\sim 1.3 \mathrm{eV}$. No magnetic anisotropy was found in the cubic $\mathrm{Co}_{2} \mathrm{TiO}_{4}$ single crystals showing a magnetic behavior similar to polycrystals. The rhombohedral $\mathrm{CoTiO}_{3}$ crystals revealed a magnetic behavior of an easy plane antiferromagnet with a significant anisotropy of the transverse magnetic susceptibilities along and perpendicular to the trigonal $c$ axis.
\end{abstract}

PACS: 75.47.Lx Magnetic oxides;

75.47.Pq Other materials.

Keywords: growth processes, titanium-cobalt-oxide, magnetic anisotropy.

\section{Introduction}

The cobalt titanates $\mathrm{Co}_{2} \mathrm{TiO}_{4}, \mathrm{CoTiO}_{3}$, and $\mathrm{CoTi}_{2} \mathrm{O}_{5}$ are a promising class of materials with many possible applications among others as new magnetodielectric materials. Lately a noticeable increase of interest for investigations of magnetic, electrical, optical properties of various Co-containing oxide compounds occurs [1-4]. But the most of physical investigations of cobalt titanates were carried out using of polycrystals or thin films. Only in few papers single crystals were investigated. In particular, highly crystalline and idiomorphic $\mathrm{CoTiO}_{3}$ single crystals with a well-defined polyhedral morphology were grown by a common flux method in Ref. 5. Pseudobrookite compounds $\mathrm{CoTi}_{2} \mathrm{O}_{5}, \mathrm{MgTi}_{2} \mathrm{O}_{5}$, and $\mathrm{FeTi}_{2} \mathrm{O}_{5}$ were prepared by solid state reactions and single crystals of quenched samples were examined by x-ray in Ref. 6 .

Possible difficulty of crystal growth from the melt is connected with complicated structure of $P-T-X$ phase dia- gram of the Co-Ti-O system [7] (Fig. 1). In addition to the $\mathrm{CoO}$ and $\mathrm{TiO}_{2}$ compounds, three cobalt titanates $\mathrm{Co}_{2} \mathrm{TiO}_{4}$, $\mathrm{CoTiO}_{3}$, and $\mathrm{CoTi}_{2} \mathrm{O}_{5}$ also exist. The two former titanates melt congruently at 1562 and $1463{ }^{\circ} \mathrm{C}$, respectively, while $\mathrm{CoTi}_{2} \mathrm{O}_{5}$ melts incongruently at $1482{ }^{\circ} \mathrm{C}$ [8]. Results of modern researches of these systems [9] show that cobalt ion valence can be change with $\mathrm{Co}_{3} \mathrm{O}_{4}$ oxide formation and depends on the temperature and the ambient gas. So real phase equilibrium in this system includes such components as $\mathrm{TiO}_{2}, \mathrm{CoO}, \mathrm{Co}_{3} \mathrm{O}_{4}, \mathrm{CoTiO}_{3}, \mathrm{Co}_{2} \mathrm{TiO}_{4}$, and $\mathrm{CoTi}_{2} \mathrm{O}_{5}$. The stabilization of $\mathrm{Co}_{2} \mathrm{TiO}_{4}$ structure and others as a single phase is more expected at high temperature and this high-temperatures state is quenched by rather quick cooling of the crystal.

The purpose of this work consisted in crystal growth of cobalt titanates because these crystals represent interest as the objects of investigation of lattice dynamic and electronic structure of spinels [10], as possible substrate materials 


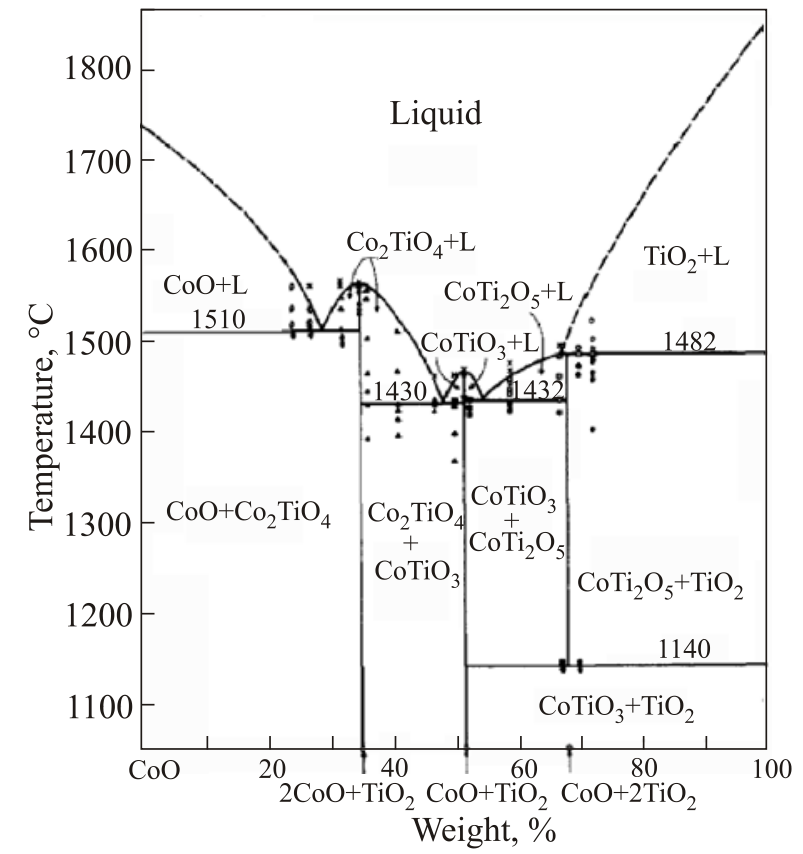

Fig. 1. Phase diagram for the system $\mathrm{CoO}-\mathrm{TiO}_{2}$ in air [7].

for magnetic ferrospinel film deposition $\left(\mathrm{Co}_{2} \mathrm{TiO}_{4}\right)$, as materials with expected notable magnetodielectric properties $\left(\mathrm{CoTiO}_{3}\right)$ [11] and investigation of peculiarities ions ordering in pseudobrookite structure $\left(\mathrm{CoTi}_{2} \mathrm{O}_{5}\right)$ [6]. The use of single crystals permits to investigate anisotropic properties of materials, to study electric transport features more precisely and perhaps to find and study magnetoelectric phenomena in these materials.

The floating zone melting method of crystal growth with light heating provides conditions for obtaining of cobalt titanate single-phase crystals. If the high-temperature annealing of single crystals in growing process is used, it is possible to obtain almost perfect crystals.

\section{Experimental details}

The single crystals have been grown by crucibleless floating zone (FZ) melting method with light heating by means of URN-2-ZM equipment [11]. Cobalt and titanium oxides of chemicals $4 \mathrm{~N}$ purity were used as starting materials. Oxide mixtures corresponding to the stoichiometric $\mathrm{Co}_{2} \mathrm{TiO}_{4}, \mathrm{CoTiO}_{3}$, and $\mathrm{CoTi}_{2} \mathrm{O}_{5}$ compositions were used as starting compounds to produce the feed rods for floating zone by common ceramic technology.

The technological conditions of $\mathrm{Co}_{2} \mathrm{TiO}_{4}, \mathrm{CoTiO}_{3}$, and $\mathrm{CoTi}_{2} \mathrm{O}_{5}$ single-crystal growth by $\mathrm{FZ}$ were as follows (almost the same for all compositions):

- Linear crystallization speed was 5-10 mm/h;

- Rotation frequency of crystal and feed rod were 40 and $1 \mathrm{r} / \mathrm{min}$, respectively;

- For $\mathrm{Co}_{2} \mathrm{TiO}_{4} \mathrm{x}$-ray oriented single crystalline seeds about $3 \times 3 \times 12 \mathrm{~mm}$ in size cut from magnetic ferrospinel crystals or from preliminary grown Co-Ti spinel crystals were used;

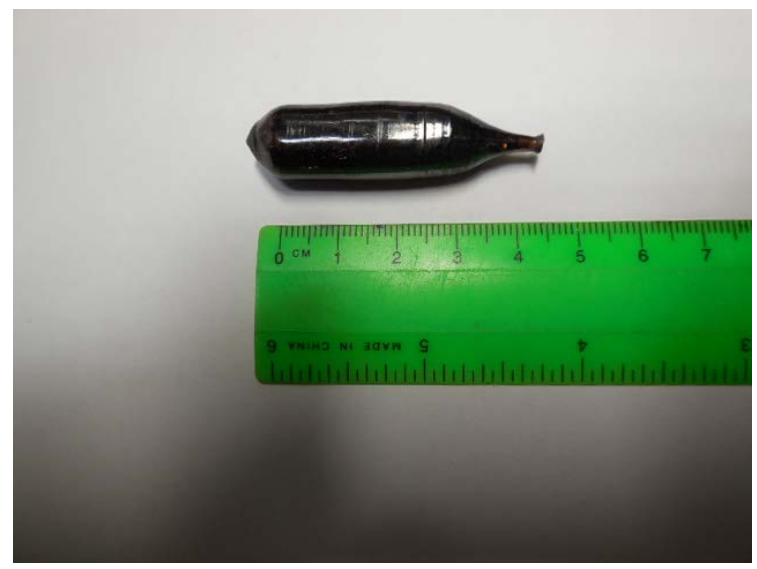

Fig. 2. Single crystal of $\mathrm{Co}_{2} \mathrm{TiO}_{4}$.

- For $\mathrm{CoTiO}_{3}$ and $\mathrm{CoTi}_{2} \mathrm{O}_{5}$ crystal growth the fragments of early grown crystals were used as a seed crystal;

- The growth was performed in an air atmosphere or in a flow of oxygen or argon;

- Annealing furnace temperature in growth processing was equal $1250{ }^{\circ} \mathrm{C}$;

- Using FZ method single crystals were obtained with a diameter of 12-15 mm and length up to $60 \mathrm{~mm}$ (Fig. 2).

The diagnostics of the samples were performed by diffraction methods (x-ray phase analysis and Laue method). Microstructure of the samples was investigated using JSM-5910LV (JEOL) scanning electron microscope in Z-contrast backscattered electrons mode. Determination of the chemical composition was carried out by means of x-ray energy dispersive analysis using AZtecENERGY (Oxford Instruments) analytical system. The X-ray analysis (XRD) of the samples was conducted using Bruker D2 Phaser powder x-ray diffractometer with $\mathrm{Cu}-K_{\alpha}$ radiation. Processing of the results and the phase analysis of samples was performed using software packages DIFFRAC ${ }^{\text {plus }}$ (EVA and TOPAS 4.2.0.2). Laue images have been obtained with digital apparatus of Photonic Science.

The magnetic properties were studied in static magnetic fields up to $5 \mathrm{~T}$ at temperatures from 2 to $340 \mathrm{~K}$ by the SQUID magnetometer (Quantum Design). The electrotransport properties were measured by means of Keithley 6517A electrometer with silver paste electrical contacts.

\section{X-ray and microstructure characterization of the grown crystals}

According to the phase diagram of the $\mathrm{CoO}-\mathrm{TiO}_{2}$ system (Fig. 1) there should not be a problem to grow from the melt the crystals of the $\mathrm{Co}_{2} \mathrm{TiO}_{4}$ and $\mathrm{CoTiO}_{3}$ compounds due to their congruent melting. On the other hand, the $\mathrm{CoTi}_{2} \mathrm{O}_{5}$ compound suffering an incongruent melting requires special conditions for a good crystal grown. Actually, very perfect $\mathrm{Co}_{2} \mathrm{TiO}_{4}$ and pseudobrookite $\mathrm{CoTi}_{2} \mathrm{O}_{5}$ crystals were grown for used FZ growth parameters that was confirmed by x-ray Laue patterns (Fig. 3), powder 


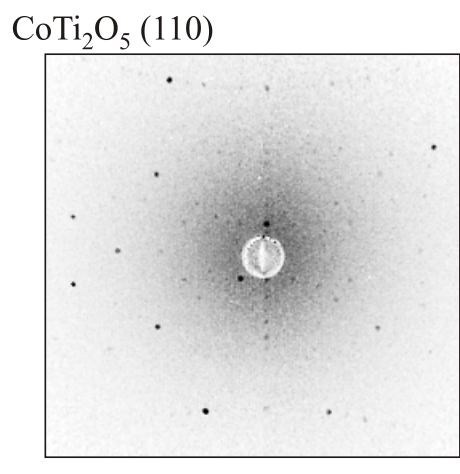

$\mathrm{Co}_{2} \mathrm{TiO}_{4}(100)$

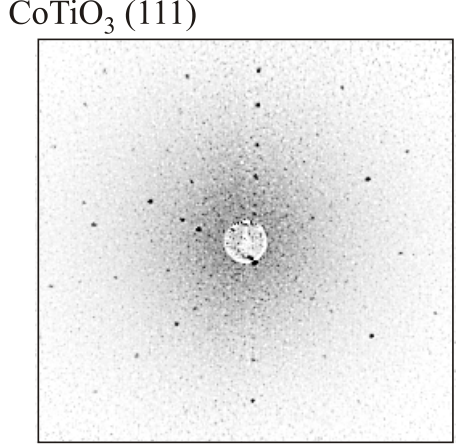

$\mathrm{CoTiO}_{3}(110)$

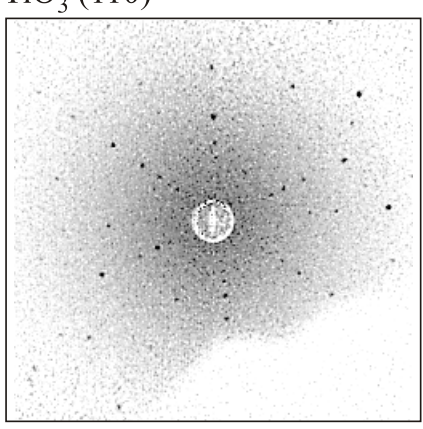

Fig. 3. Laue patterns of the grown crystals Co-Ti oxide compounds.

(Fig. 4) and single-crystal (Fig. 5) diffraction measurements. Small angular width $\left(\sim 0.08-0.1^{\circ}\right)$ of representing $\mathrm{x}$-ray reflections for polished cross sections of the grown single crystals confirms their high quality and low values of internal stress distortions (Fig. 5).

In spite of good Laue pattern we found that $\mathrm{CoTiO}_{3}$ crystals contain small amount of second phase inclusion (Fig. 6). This is connected with a complicated behavior of the compounds at high temperatures [8] and high temperature gradients in cross section of melts at a growth interface. It results in a decomposition of main $\mathrm{CoTiO}_{3}$ compound and appearance of two types of secondary phases: $\mathrm{Co}_{2} \mathrm{TiO}_{4}$ and $\mathrm{CoTi}_{2} \mathrm{O}_{5}$. The $\mathrm{Co}_{2} \mathrm{TiO}_{4}$ secondary phase precipitates at a periphery of a crystal cross section while $\mathrm{CoTi}_{2} \mathrm{O}_{5}$ one precipitates in a central part. The volume of

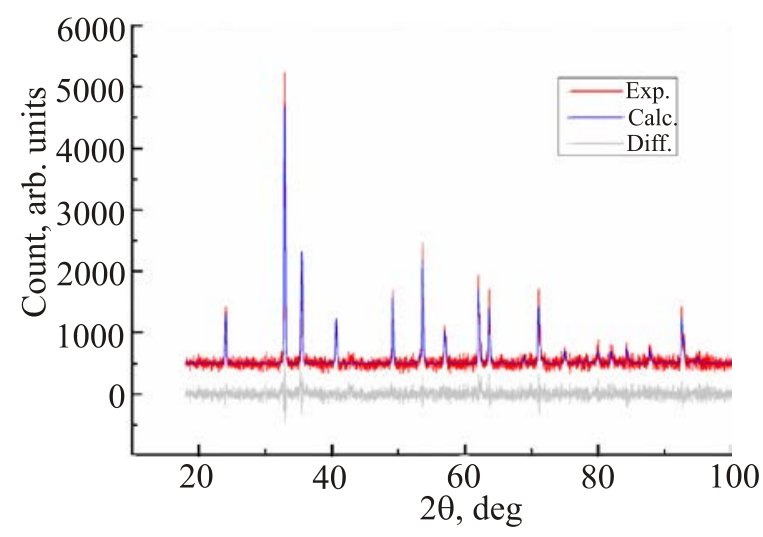

Fig. 4. (Color online) Refined x-ray diffraction pattern for $\mathrm{CoTiO}_{3}$ powder. second phases is less $3 \%$ and is not reflected in $\mathrm{x}$-ray measuring data. The quality of the $\mathrm{Co}_{2} \mathrm{TiO}_{4}$ and $\mathrm{CoTi}_{2} \mathrm{O}_{5}$ crystals almost do not depend on a gas ambient during a crystal growth. The amount of the secondary phases decreases but does not disappear completely during the $\mathrm{CoTiO}_{3}$ growth in an oxygen flow. This observation con-
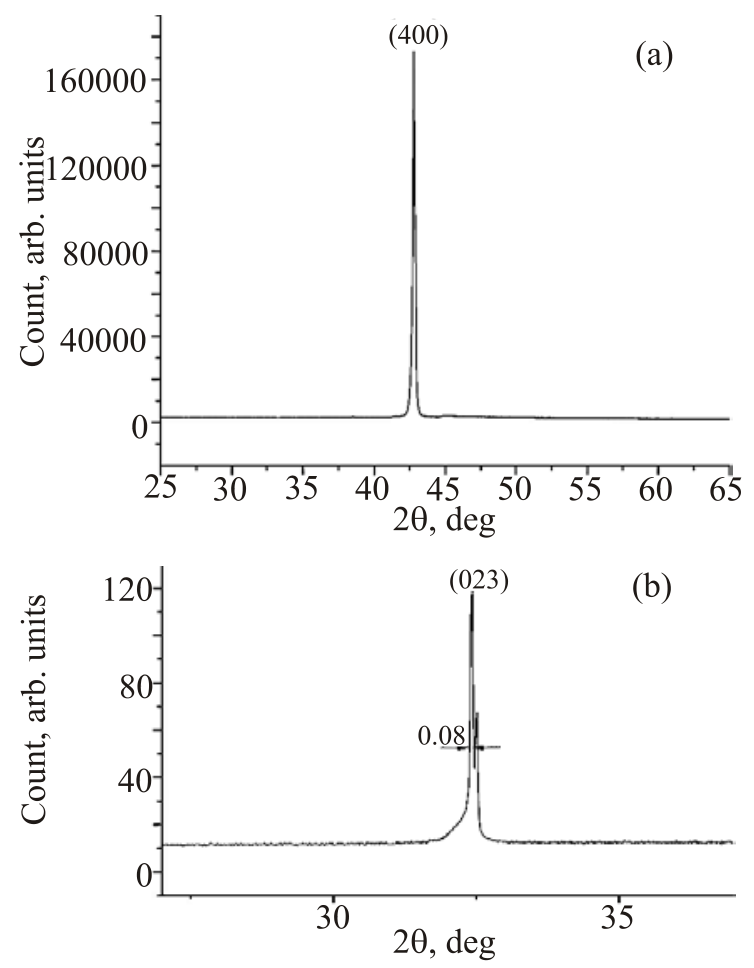

Fig. 5. Single crystalline $\mathrm{x}$-ray diffraction patterns of the cross section of $\mathrm{Co}_{2} \mathrm{TiO}_{4}$ (a) and $\mathrm{CoTi}_{2} \mathrm{O}_{5}$ (b) single crystals. 


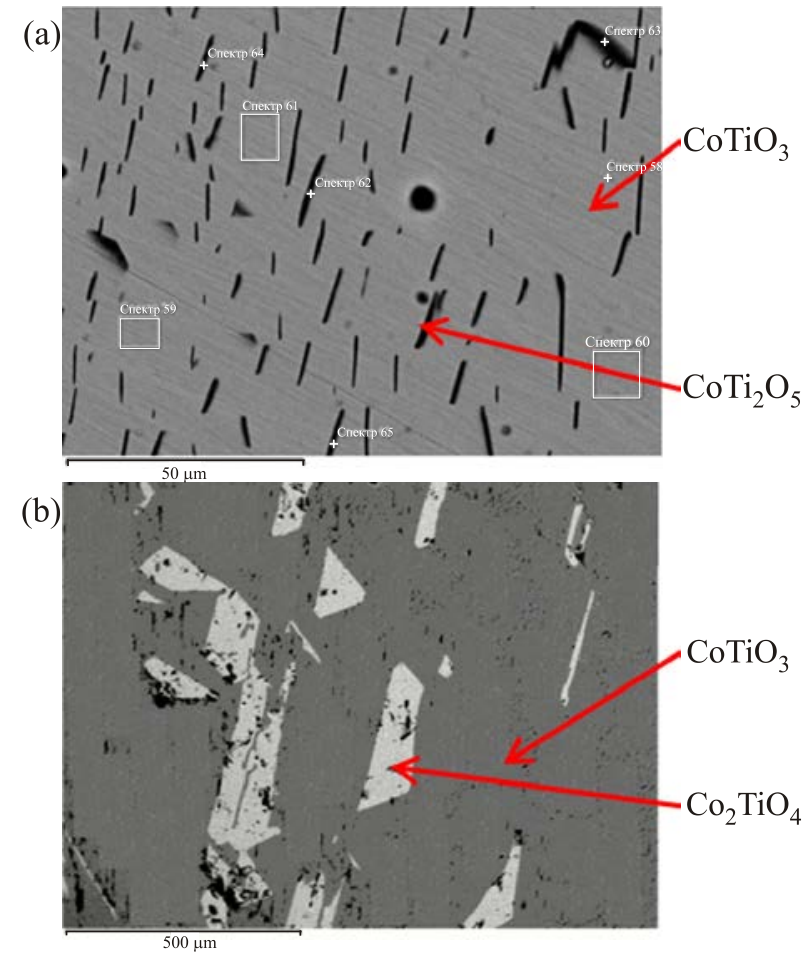

Fig. 6. SEM backscattered electron image (Z-contrast) in peripheral (a) and central (b) regions of the $\mathrm{CoTiO}_{3}$, grown in nonoptimal conditions.

tradicts to the phase diagram of the system according to which this compound melts congruently (Fig. 1).

The refined unit-cell parameters of the grown crystals are presented in Table 1 which demonstrates a good agreement with corresponding literature data for these compounds.

\section{Physical properties of crystals}

\subsection{Electrical resistivity of $\mathrm{Co}_{2} \mathrm{TiO}_{4}$}

As can be seen from the Fig. 7(a), the sample has enough high resistivity $\sim 10^{9} \mathrm{Ohm} \cdot \mathrm{cm}$ at room temperature and close to ohmic contacts. With decreasing temperature the resistance grows exponentially versus $1 / T$ (Fig. 7(b)) and gives the forbidden zone (bandgap) value of $\sim 1.3 \mathrm{eV}$. Below $\sim 200 \mathrm{~K}$ the resistance of crystal becomes very high as in good isolators and its measurement becomes impossible. We also tried to find some manifestations of magnetoelectric effects in the temperature and magnetic field dependences of pyroelectric current along [100] axis in magnetic field $\mathbf{H}$ perpendicular to electric field $\mathbf{E}$ but these attempts were failed.

\subsection{Magnetic properties of $\mathrm{Co}_{2} \mathrm{TiO}_{4}$}

Results of our magnetic measurement are in a good agreement with data of Ref. 1 . The found values of ferrimagnetic ordering temperature ( $48 \pm 1) \mathrm{K}$ and compensation point $(30.5 \pm 1) \mathrm{K}$ are close to the corresponding values of $T_{N} \sim 47.8 \mathrm{~K}$ and $T_{\text {comp }} \sim 32 \mathrm{~K}$ from Ref. 1 . Temperature dependences of magnetization at different magnetic fields and magnetization curves at different temperatures also practically coincided with the data obtained in [1] (Figs. 8-10). However we obtained another values of the asymptotic Curie point $T_{a}$, and the effective magnetic moment $\mu_{\mathrm{eff}}$ determined from the temperature dependence of the inverse magnetic susceptibility (Fig. 9), namely, $T_{a}=(-150 \pm 2) \mathrm{K}$ and $\mu_{\text {eff }}=(7.0 \pm 0.1) \mu_{B}$ according to our data in contrast to $T_{a}=-125 \mathrm{~K}$ and $\mu_{\mathrm{eff}}=6.5 \mu_{B}$ of Ref. 1. Such discrepancy for the values of the effective moment provokes doubts for the reliability of the Co ion moments for different crystallographic positions found in Ref. 1 .

Magnetization curves linearly depend on magnetic field $H$ at the compensation point $30 \mathrm{~K}$, but they exhibit hysteresis loops below and above this point (Figs. 10(a)-(c)). Near Curie point the hysteresis becomes narrow and disappears in paramagnetic state (Figs. 10(c), (d)). Most importantly, we haven't found noticeable magnetic anisotropy along different crystallographic directions of the cubic crystal: $<100>,<110>$, and $<111>$ in any magnetic state. Possible explanation of this phenomenon can be connected with low anisotropy above and near $T_{N}$ and appearance of a spinglass state at low temperatures.

\subsection{Magnetic properties of $\mathrm{CoTiO}_{3}$}

In contrast to the almost isotropic $\mathrm{Co}_{2} \mathrm{TiO}_{4}$ the $\mathrm{CoTiO}_{3}$ crystal, possessing rhombohedral (R3) ilmenite structure, exhibits a significant anisotropy of the magnetic characteristics up to temperatures of $~ 350 \mathrm{~K}$ (Fig. 11) in a good agreement with Ref. 12. The dc magnetic susceptibility $\chi_{d c}=\sigma / H$ along the trigonal $c$ axis is noticeably smaller than that in the basis plane, within which the magnetic anisotropy is small. The Néel temperature $T_{N}=37-38 \mathrm{~K}$ clearly emerges as a maximum in the temperature

Table 1. Unit-cell parameters in comparison with literature data

\begin{tabular}{c|c|c|c|c|c}
\hline \hline Composition & $a, \AA$ & $b, \AA$ & $c, \AA$ & Space group & JCPDS, card No \\
\hline \hline $\mathrm{Co}_{2} \mathrm{TiO}_{4}$ & $8.4570(3)$ & & & $F d 3 m$ & Refined \\
\hline & 8.434 & & & $R 3$ & $39-1410$ \\
\hline $\mathrm{CoTiO}_{3}$ & $5.0662(6)$ & & $13.916(2)$ & & Refined \\
\hline & 5.056 & & 13.91 & & $77-1356$ \\
\hline $\mathrm{CoTi}_{2} \mathrm{O}_{5}$ & $3.7319(7)$ & $9.721(2)$ & $10.094(3)$ & Cmcm & Refined \\
\hline & 3.732 & 9.718 & 10.06 & & $76-1600$ \\
\hline \hline
\end{tabular}



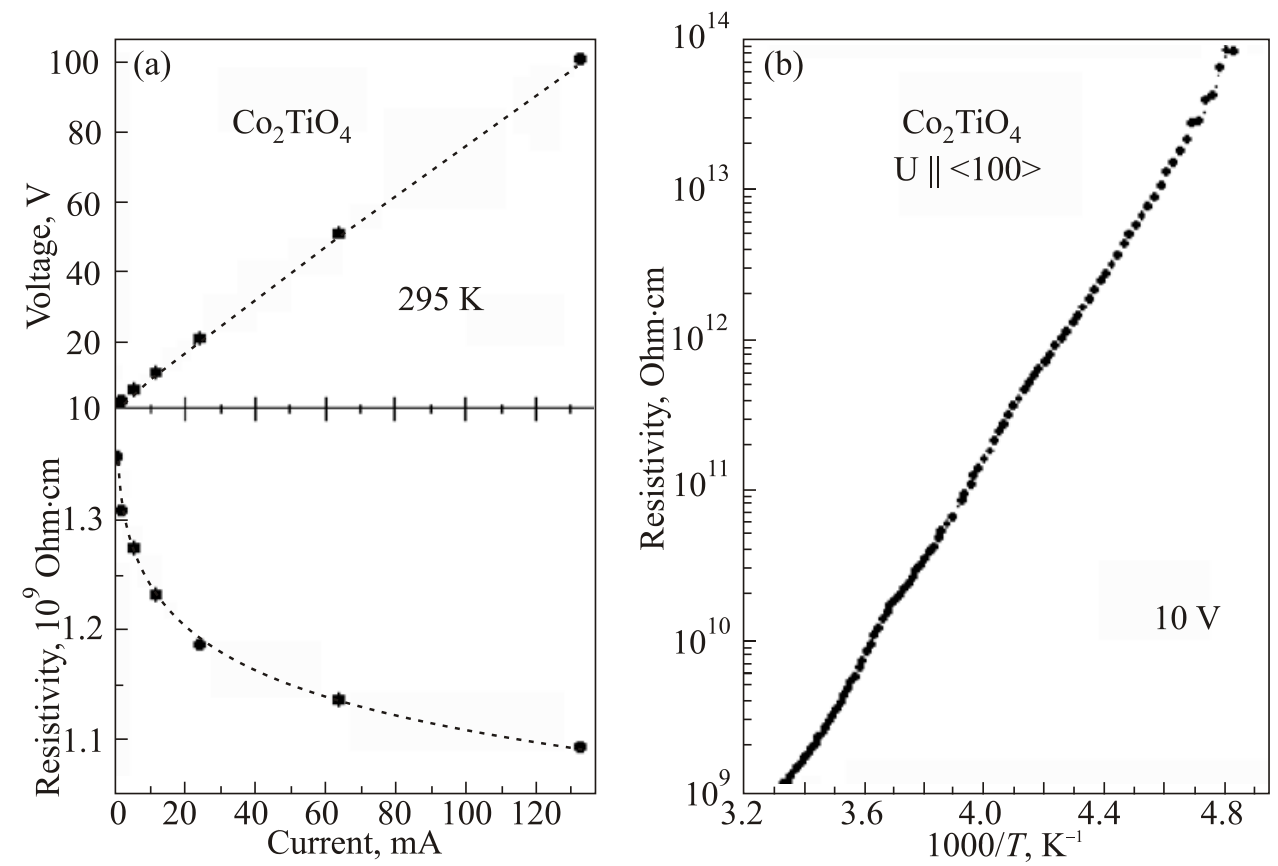

Fig. 7. Electric properties of the $\mathrm{Co}_{2} \mathrm{TiO}_{3}$ crystals: (a) Current-voltage characteristic (top) and dependence of the electric resistivity on current along the $<100>$ crystallographic direction at $T=295 \mathrm{~K}$; (b) Temperature dependence of electrical resistivity measured along the $<100>$ axis in the applied voltage $10 \mathrm{~V}$.

dependence of $\chi_{d c}(T)$. A blurred nature of the maximum for the $\chi_{d c}(T)$ for $\mathbf{H} \| \mathbf{c}$ is associated with the presence of impurity phases of $\mathrm{Co}_{2} \mathrm{TiO}_{4}$ and $\mathrm{CoTi}_{2} \mathrm{O}_{5}$ which also possess anomalies of magnetic properties at similar temperatures. Magnetization curves along $c$ axis are linear up to $5 \mathrm{~T}$ at all temperatures from 1.9 to $340 \mathrm{~K}$, however, in a perpendicular direction (i.e., in the basis plane) the $\sigma(H)$

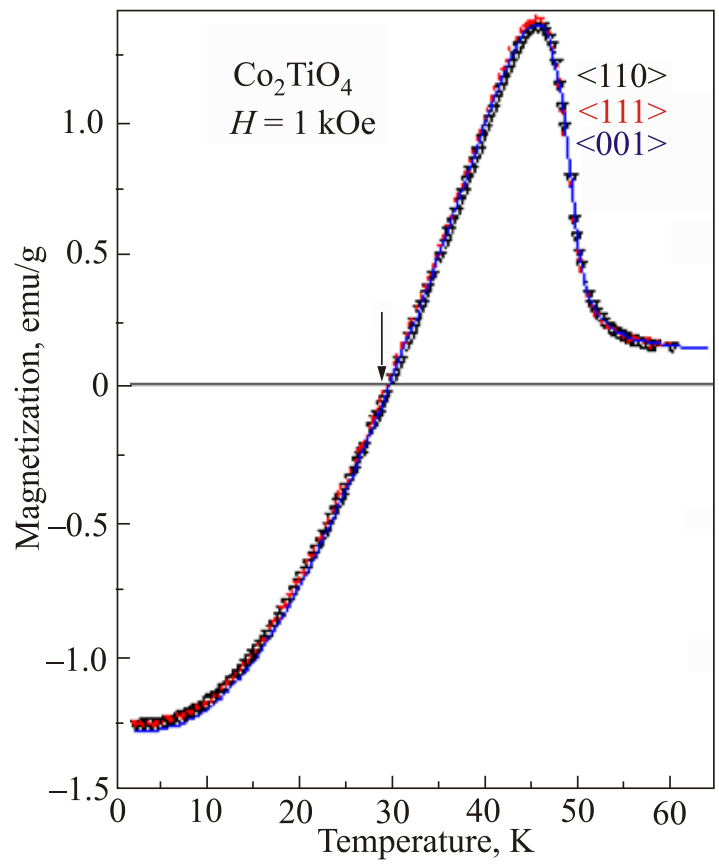

Fig. 8. (Color online) Temperature dependences of magnetization measured in the field of 1 kOe along different crystallographic directions. curves become significantly nonlinear in the fields of 3-4 T at $T<T_{N}$ thus indicating on a rotation of the $\mathrm{Co}^{2+}$ magnetic moments in the basal plane to the direction perpendicular to the applied field (inset in Fig. 11). In a whole $\mathrm{CoTiO}_{3}$ shows up properties of an easy-plane antiferromagnet possessing a significant anisotropy of the transverse magnetic susceptibilities along and perpendicular to the $c$ axis which

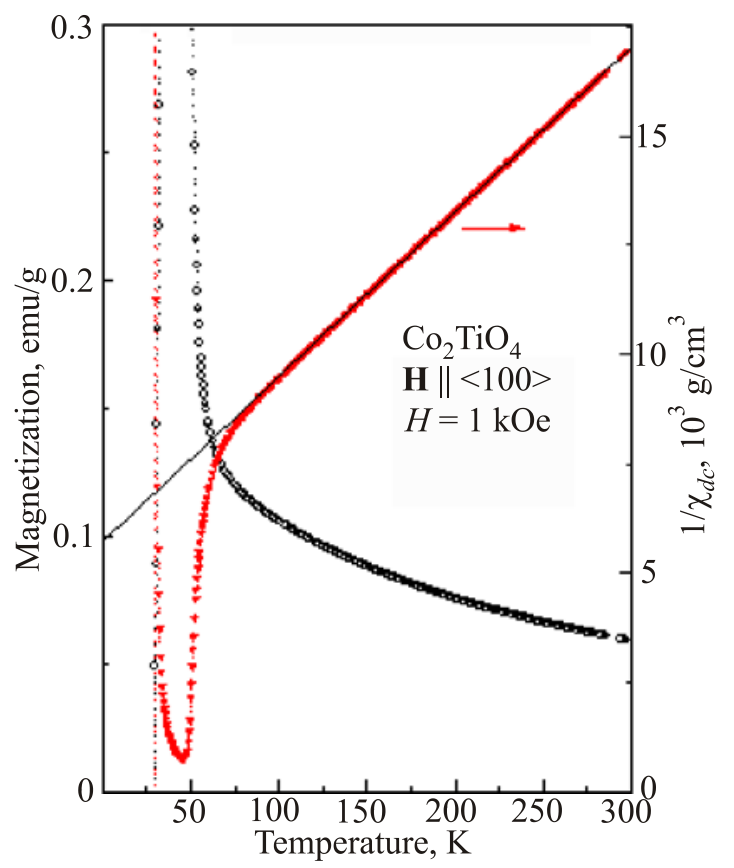

Fig. 9. (Color online) Temperature dependences of the magnetization along $<100>$ axis in $H=1 \mathrm{kOe}$ (left scale) and reverse magnetic susceptibility (right scale). 

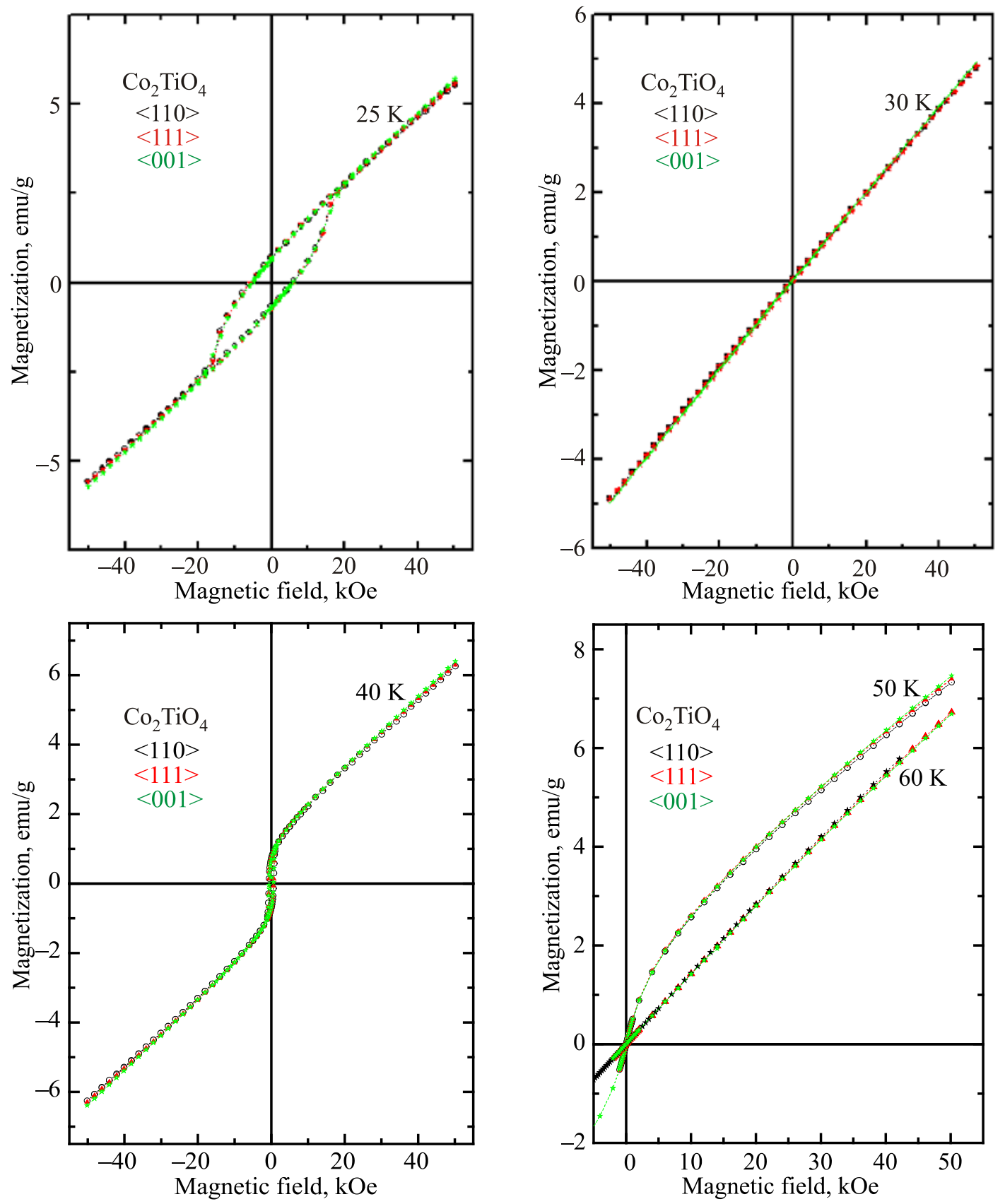

Fig. 10. (Color online) Magnetization curves of $\mathrm{Co}_{2} \mathrm{TiO}_{3}$ single crystals along $<110>,<111>$, $<001>$ pseudocubic crystallographic directions and various temperatures: (a) $T=25 \mathrm{~K}$, (b) $30 \mathrm{~K}$, (c) $40 \mathrm{~K}$ and (d) 50 and $60 \mathrm{~K}$.

origin could be related to a noticeable anisotropy of $g$ factor of the $\mathrm{Co}^{2+}$ ions with a strong spin-orbital coupling.

\section{Conclusion}

Single crystals of titanium cobaltites $\mathrm{Co}_{2} \mathrm{TiO}_{4}, \mathrm{CoTiO}_{3}$, and $\mathrm{CoTi}_{2} \mathrm{O}_{5}$ have been grown for the first time by floating zone melting with light heating in different gas ambient. Their crystal structure parameters were investigated by x-ray diffraction and electron microscopy. It was established that rather perfect $\mathrm{Co}_{2} \mathrm{TiO}_{4}$ and $\mathrm{CoTi}_{2} \mathrm{O}_{5}$ single crystals can be grown in air with further annealing at $1250{ }^{\circ} \mathrm{C}$. However, independently of gas ambient the $\mathrm{CoTiO}_{3}$ crystals contain precipitation of second phases in amount of $\sim 1 \%$, representing either $\mathrm{CoTi}_{2} \mathrm{O}_{5}$ in the central part of a boule or $\mathrm{Co}_{2} \mathrm{TiO}_{4}$ in its periphery part.

$\mathrm{Co}_{2} \mathrm{TiO}_{4}$ exhibits semiconducting behavior with the energy gap of $\sim 1.3 \mathrm{eV}$. No magnetic anisotropy was found in $\mathrm{Co}_{2} \mathrm{TiO}_{4}$ single crystals in a wide temperature range and their magnetic behavior was similar to polycrystalline samples. In spite of presence of a small amount of impurity phases, temperature and field dependences of magnetization in the grown $\mathrm{CoTiO}_{3}$ crystal adequately reflect the be- 


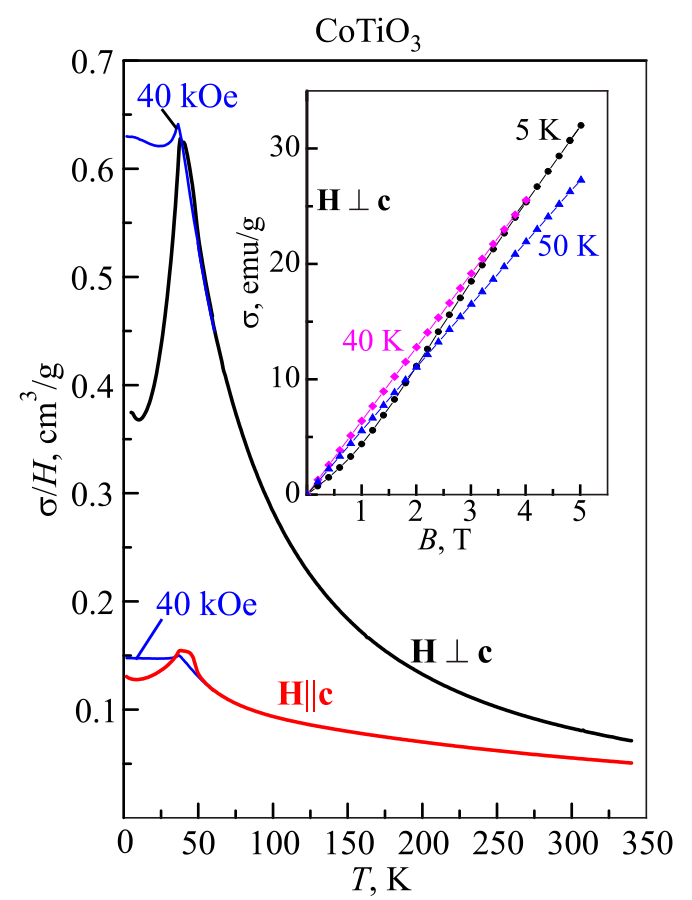

Fig. 11. (Color online) Temperature dependences of dc magnetic susceptibility measured for $1 \mathrm{kOe}$ along and perpendicular to the trigonal $c$ axis. In the low-temperature region the data for $H=40 \mathrm{kOe}$ are also presented. The inset shows the magnetization curves at different temperatures at $\mathbf{H} \perp \mathbf{c}$.

havior of pure $\mathrm{CoTiO}_{3}$ easy-plane antiferromagnetic phase below $37 \mathrm{~K}$ exhibiting a remarkable anisotropy of the transverse magnetic susceptibilities.

The work is supported by the Russian Scientific Foundation (project 16-12-10531).
1. S. Nayak, S. Thota, D.C. Joshi, M. Krautz, A. Waske, A. Behler, J. Eckert, T. Sarkar, M. S. Andersson, R. Mathieu, V. Narang, and M.S. Seehra, Phys. Rev. B 92, 214434 (2015).

2. S. Nayak, K. Dasari, D.C. Joshi, P. Pramanik, R. Palai, A. Waske, R.N. Chauhan, N. Tiwari, T. Sarkar, and S. Thota, J. Appl. Phys.120, 163905 (2016).

3. T. Acharya and R.N.P. Choudhary, Mater. Chem. Phys. 177, 131 (2016).

4. Jaye K. Harada, Luke Balhorn, Josef Hazi, Moureen C. Kemei, and Ram Seshadri, Phys. Rev. B 93, 104404 (2016).

5. Q. Wang, Q. Guo, L. Wang, and B. Li, Dalton Transactions 45, 17748 (2016).

6. A. Yankin, O. Vikhreva, and V. Balakirev, J. Phys. Chem. Solids 60, 139 (1999).

7. H. Muller-Buschbaum and M. Waburg, Monatshefte fur Chemie 114, 21 (1983).

8. B. Brežnỳ and A. Muan, J. Inorg. Nucl. Chem. 31, 649 (1969).

9. K.T. Jacob and G. Rajitha, J. Chem. Thermodynamics 42, 879 (2010).

10. M.A. Prosnikov, A.D. Molchanov, R.M. Dubrovin, K.N. Boldyrev, A.N. Smirnov, Yu. Davydov, A.M. Balbashov, M.N. Popova, and R.V. Pisarev, Phys. Solid State 58, 2516 (2016).

11. A.M. Balbashov and S.K. Egorov, J. Cryst. Growth 52, 498 (1981).

12. H. Watanabe, H. Yamauchi, and H. Takei, J. Magn. Magn. Mater. 15-18, 549 (1980). 